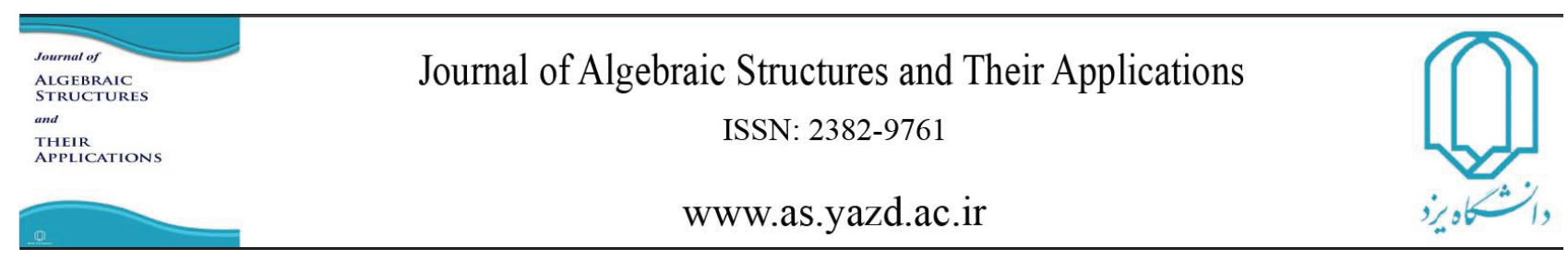

Algebraic Structures and Their Applications Vol. 5 No. 1 ( 2018 ) pp 69-80.

\title{
ON ENDO-SEMIPRIME AND ENDO-COSEMIPRIME MODULES
}

\author{
P. KARIMI BEIRANVAND AND R. BEYRANVAND*
}

Communicated by M.A. Iranmanesh

\begin{abstract}
In this paper, we study the notions of endo-semiprime and endocosemiprime modules and obtain some related results. For instance, we show that in a right self-injective ring $R$, all nonzero ideals of $R$ are endo-semiprime as right (left) $R$-modules if and only if $R$ is semiprime. Also, we prove that both being endo-semiprime and being endo-cosemiprime are Morita invariant properties.
\end{abstract}

\section{INTRODUCTION}

Throughout this paper, all rings have identity elements and all modules are right unitary. Unless otherwise stated, $R$ denotes an arbitrary ring with identity element. If $M$ is a right (resp., left) $R$-module, we use the notation $M_{R}$ (resp., $\left.{ }_{R} M\right)$. Let $M$ be an $R$-module. If $N$ is a submodule of $M$, we write $N \leq M$ and the annihilator of $N$ (in $R$ ) is denoted by $\operatorname{ann}_{R}(N)=\{r \in R \mid N r=0\}$. Also, $N \leq M$ is called a fully invariant submodule of $M$ if for every $R$-endomorphism $f: M \rightarrow M, f(N) \subseteq N$.

DOI :http://dx.doi.org/10.29252/asta.5.1.69

MSC(2010): 16D10, 16D90, 16N60.

Keywords: Endo-prime modules; endo-semiprime modules; endo-coprime modules; endo-cosemiprime modules.

Received: 15 February 2018, Accepted: 29 September 2018

*Corresponding author

(C) 2018 Yazd University. 
An $R$-module $M$ is said to be quasi injective if for any submodule $N$ of $M$, any $R$ homomorphism from $N$ to $M$ can be extended to an endomorphism of $M$. A proper ideal $P$ of a ring $R$ is called a prime ideal of $R$ if for any two ideals $I$ and $J$ of $R, I J \subseteq P$ implies that $I \subseteq P$ or $J \subseteq P$. Also, $P$ is called a semiprime ideal of $R$ if for any ideal $I$ of $R$, $I^{2} \subseteq P$ implies that $I \subseteq P$. The notion of prime ideals was extended from rings to modules by Dauns in [3]. In fact, a nonzero $R$-module $M$ is called prime if $\operatorname{ann}_{R}(M)=\operatorname{ann}_{R}(N)$, for every nonzero submodule $N$ of $M$. Also, a nonzero $R$-module $M$ is called semiprime if $\operatorname{ann}_{R}(N)$ is a semiprime ideal of $R$, for any nonzero submodule $N$ of $M$. By a prime (resp., semiprime) submodule of a module $M$ we mean a submodule $N$ such that the module $M / N$ is prime (resp., semiprime). It is easy to see that a proper submodule $N$ of $M$ is a prime submodule if for any submodule $K$ of $M$ and any ideal $I$ of $R, K I \subseteq N$ implies that $K \subseteq N$ or $M I \subseteq N$. The dual of prime modules was introduced and studied by Ceken, Alkan and smith in [2]. In fact, a nonzero $R$-module $M$ is called coprime if $\operatorname{ann}_{R}(M)=\operatorname{ann}_{R}(M / N)$, for any proper submodule $N$ of $M$. Also, a nonzero $R$-module $M$ is called cosemiprime if $\operatorname{ann}_{R}(M / N)$ is a semiprime ideal of $R$, for any proper submodule $N$ of $M$. It is easy to see that every prime (resp., coprime) $R$-module is semiprime (resp., cosemiprime). More details about these notions can be found in $[1, \underline{6}, \mathbf{8}]$.

Let $M$ be a right $R$-module and $S=\operatorname{End}\left(M_{R}\right)$. In [5], the authors introduced and studied the notion of endo-prime modules. In fact, $M$ is called endo-prime if for any nonzero fully invariant submodule $N$ of $M$ and any $f \in S, f N=0$ implies that $f=0$, i.e., $\operatorname{ann}_{S}(N)=0$, for any nonzero fully invariant submodule $N$ of $M$. In this paper, we generalize this notion as follows: we say that $M$ is endo-semiprime if $\operatorname{ann}_{S}(N)$ is a semiprime ideal of $S$ for any nonzero fully invariant submodule $N$ of $M$. Also, we introduce and study the dual notion of endo-semiprime. A nonzero right $R$-module $M$ is called endo-cosemiprime if $\operatorname{ann}_{S}(M / N)$ is a semiprime ideal of $S$, for any proper fully invariant submodule $N$ of $M_{R}$. Among other results, we prove that if $M_{R}$ is epi-retractable (resp., co-mono-retractable) such that $S=$ $\operatorname{End}\left(M_{R}\right)$ is a semiprime ring, then $M_{R}$ is endo-semiprime (resp., endo-cosemiprime) (Proposition [.J]). Also, it is shown that every semisimple module is both endo-semiprime and endo-cosemiprime (Corollary [2.14).

\section{Endo-semiprime and endo-cosemiprime modules}

Definition 2.1. Let $M$ be a nonzero right $R$-module and $S=\operatorname{End}\left(M_{R}\right)$.

(a) $M$ is called endo-semiprime if for any nonzero fully invariant submodule $N$ of $M$, $\operatorname{ann}_{S}(N)$ is a semiprime ideal of $S$. 
Alg. Struc. Appl. Vol. 5 No. 1 (2018) 69-80.

(b) $M$ is called endo-cosemiprime if for any proper fully invariant submodule $N$ of $M$, $\operatorname{ann}_{S}(M / N)$ is a semiprime ideal of $S$.

For example, if the integer number $n$ is square-free, then $\mathbb{Z} / n \mathbb{Z}$ as $\mathbb{Z}$-module is endosemiprime. Because $n \mathbb{Z}$ is a semiprime ideal of $\mathbb{Z}$, see Corollary [2.7. Also, from the above definition, we can easily see that if $M_{R}$ is endo-semiprime, then $S=\operatorname{End}\left(M_{R}\right)$ is a semiprime ring. We will show that $\mathbb{Z}_{p^{\infty}}$ is endo-cosemiprime while is not endo-semiprime, see Example [2.13. Also, it is easy to see that if $M_{R}$ is endo-cosemiprime, then $S=\operatorname{End}\left(M_{R}\right)$ is a semiprime ring.

Proposition 2.2. Let $M$ be a right $R$-module and $S=\operatorname{End}\left(M_{R}\right)$. Then $M_{R}$ is endo-semiprime (resp., endo-cosemiprime) if and only if ${ }_{S} M$ is a semiprime (resp., cosemiprime) module.

Proof. First suppose that $M_{R}$ is endo-semiprime and $0 \neq N \leq{ }_{S} M$. Then $N R$ is a fully invariant submodule of $M$ and by hypothesis, $\operatorname{ann}_{S}(N R)$ is a semiprime ideal of $S$. Clearly, $\operatorname{ann}_{S}(N R)=\operatorname{ann}_{S}(N)$ and this shows that ${ }_{S} M$ is semiprime. Conversely, if ${ }_{S} M$ is semiprime, then it is clear that $M_{R}$ is endo-semiprime.

Now, let $M_{R}$ be an endo-cosemiprime module and $K$ be a proper submodule of ${ }_{S} M$. We set $J=\operatorname{ann}_{S}(M / K)$. Then $J M \subseteq K \varsubsetneqq S M$ and so $J M$ is a proper fully invariant submodule of $M_{R}$. Since $M_{R}$ is endo-cosemiprime, $\operatorname{ann}_{S}(M / J M)$ is semiprime. On the other hand, we have $J \subseteq \operatorname{ann}_{S}(M / J M) \subseteq \operatorname{ann}_{S}(M / K)=J$. Thus, $\operatorname{ann}_{S}(M / J M)=\operatorname{ann}_{S}(M / K)$ is semiprime and hence, ${ }_{S} M$ is cosemiprime. Conversely, if $K$ is a proper fully invariant submodule of $M_{R}$, then $K$ is a proper submodule of ${ }_{S} M$ and so by assumption $\operatorname{ann}_{S}(M / K)$ is semiprime.

We need the following lemmas.

Lemma 2.3. Let $M$ be a right $R$-module and $I$ be an ideal of $R$ such that $M I=0$. Then

(1) $\operatorname{End}\left(M_{R}\right)=\operatorname{End}\left(M_{R / I}\right)$;

(2) $M_{R}$ is endo-semiprime if and only if $M_{R / I}$ is endo-semiprime;

(3) For any submodule $N$ of $M$, $\operatorname{ann}_{R}(N)$ is a semiprime ideal in $R$ if and only if $\operatorname{ann}_{R / I}(N)$ is a semiprime ideal in $R / I$.

Proof. (1) Since $m r=m(r+I)$, for any $r \in R$ and $m \in M$, we have $\operatorname{End}\left(M_{R}\right)=\operatorname{End}\left(M_{R / I}\right)$. (2) For any $N \subseteq M$, we have $N$ is a fully invariant submodule of $M_{R}$ if and only if $N$ is a fully invariant submodule of $M_{R / I}$. Now, the result follows from part (1).

(3) We first assume that $\operatorname{ann}_{R}(N)$ is a semiprime ideal in $R$, where $N$ is a submodule of $M_{R}$. Let $a \in R$ and $(a+I) R / I(a+I) \subseteq \operatorname{ann}_{R / I}(N)$. Then $N(a+I) R / I(a+I)=0$ and so $N(a+I)(r+I)(a+I)=0$, for any $r \in R$. Thus $N($ ara $+I)=N a r a=0$, for any $r \in R$. This

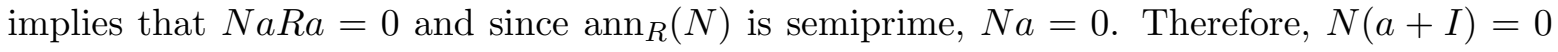


and so $a+I \in \operatorname{ann}_{R / I}(N)$. Thus $\operatorname{ann}_{R / I}(N)$ is a semiprime ideal in $R / I$. The argument for the converse is similar.

Lemma 2.4. Let $M$ be a right $R$-module and $I$ be an ideal of $R$ such that $M I=0$. Then

(1) $M_{R}$ is endo-cosemiprime if and only if $M_{R / I}$ is endo-cosemiprime;

(2) For any submodule $N$ of $M, \operatorname{ann}_{R}(M / N)$ is a semiprime ideal in $R$ if and only if $\operatorname{ann}_{R / I}(M / N)$ is a semiprime ideal in $R / I$.

Proof. By the equality $\operatorname{End}\left(M_{R}\right)=\operatorname{End}\left(M_{R / I}\right),(1)$ is clear.

For see (2), we first assume that $\operatorname{ann}_{R}(M / N)$ is a semiprime ideal in $R$, where $N$ is a submodule of $M_{R}$. Let $a \in R$ and $(a+I) R / I(a+I) \subseteq \operatorname{ann}_{R / I}(M / N)$. Then $M / N(a+I) R / I(a+I)=0$ and so $M(a+I)(r+I)(a+I) \subseteq N$, for any $r \in R$. Thus $M(\operatorname{ara}+I)=\operatorname{Mara} \subseteq N$, for

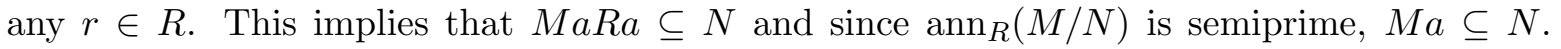
Therefore, $M(a+I) \subseteq N$ and so $a+I \in \operatorname{ann}_{R / I}(M / N)$. Thus $\operatorname{ann}_{R / I}(M / N)$ is a semiprime ideal in $R / I$. The argument for the converse is similar.

For any two non-empty subsets $A$ and $B$ of a ring $R$, we denote the set $\{r \in R \mid r A \subseteq B\}$ by $(A: B)_{l}$.

Lemma 2.5. Let $I$ be a proper right ideal in a ring $R$. Then the cyclic right $R$-module $R / I$ is endo-semiprime (resp., endo-cosemiprime) if and only if for any right ideal $J$ that properly contains $I$ and $(I: I)_{l} \subseteq(J: J)_{l}$ the following holds, for any $r \in R$ :

$$
\begin{gathered}
r(I: I)_{l} r \subseteq(J: I)_{l} \Rightarrow r \in(J: I)_{l} . \\
\left(\text { resp., } r(I: I)_{l} r \in(R: J)_{l} \Rightarrow r \in(R: J)_{l}\right) .
\end{gathered}
$$

Proof. It is easy to see that:

(1) $\operatorname{End}\left((R / I)_{R}\right) \cong(I: I)_{l} / I$.

(2) A submodule $J / I$ of the right $R$-module $R / I$ is fully invariant if and only if $(I: I)_{l} \subseteq(J$ : $J)_{l}$.

Now, suppose that $(R / I)_{R}$ is endo-semiprime and $I \varsubsetneqq J$ is a right ideal of $R$ such that $(I: I)_{l} \subseteq(J: J)_{l}$. By $(2)$ in the above, $J / I$ is a fully invariant submodule of $(R / I)_{R}$. Then its left annihilator in $\operatorname{End}\left((R / I)_{R}\right)$ is semiprime and so is in the ring $(I: I)_{l} / I$ by $(1)$. This implies that for any $r+I \in(I: I)_{l} / I$, if $\left((r+I) \frac{(I: I)_{l}}{I}(r+I)\right) J / I=0$, then $(r+I) J / I=0$. In other words; if $\left(r(I: I)_{l} r\right) J \subseteq I$, then $r J \subseteq I$. Conversely, let $J / I$ be a fully invariant submodule in $(R / I)_{R}$. Then by $(2),(I: I)_{l} \subseteq(J: J)_{l}$ and by the relation $(*)$, the left annihilator of $J / I$ is semiprime in $(I: I)_{l} / I$. So $(R / I)_{R}$ is endo-semiprime. For endo-cosemiprime, the proof is similar to the first part. 
Alg. Struc. Appl. Vol. 5 No. 1 (2018) 69-80.

Proposition 2.6. Let $I$ be a proper ideal in a ring $R$.

(1) If $(R / I)_{R}$ is endo-semiprime, then for any ideal $J$ in $R$ that properly contains $I,(J: I)_{l}$ is a semiprime ideal in $R$.

(2) $(R / I)_{R}$ is endo-cosemiprime if and only if any ideal $J$ that contains $I$, is semiprime.

Proof. (1) Let $I \varsubsetneqq J$ be an ideal of $R$ and $(R / I)_{R}$ be endo-semiprime. Then by Lemma 2.3], $(R / I)_{R / I}$ is endo-semiprime and so $\operatorname{ann}_{S}(J / I)$ is a semiprime ideal of $S$, where $S=$ $\operatorname{End}\left((R / I)_{R / I}\right)$. Since $\operatorname{End}\left((R / I)_{R / I}\right) \cong R / I$, we have $\operatorname{ann}_{R / I}(J / I)$ is a semiprime ideal of $R / I$. Again by Lemma [2.3], $\operatorname{ann}_{R}(J / I)=(J: I)_{l}$ is a semiprime ideal of $R$.

(2) It is an easy consequence of the Lemma [2.4 and this fact that $\operatorname{End}\left(R_{R}\right) \cong R$, for any ring $R$.

Corollary 2.7. Let $I$ be a proper ideal in a ring $R$. Then $I$ is semiprime if and only if $(R / I)_{R}$ is endo-semiprime.

Proof. If $(R / I)_{R}$ is endo-semiprime, then by setting $J=R$, in Proposition [2.6, we have $(R: I)_{l}=\{r \in R \mid r R \subseteq I\}=I$ is a semiprime ideal of $R$. Conversely, let $I$ be semiprime and $J$ be a right ideal in $R$ such that properly contains $I$ and $(I: I)_{l} \subseteq(J: J)_{l}$. Since $(I: I)_{l}=R$, we have $(J: J)_{l}=R$ and hence, $J$ is a two-sided ideal of $R$. Now, suppose that $\left(r(I: I)_{l} r\right) J \subseteq I$. Then $r R r J \subseteq I$ and since $J$ is two-sided ideal, we have $\operatorname{Rr} R J \operatorname{Rr} R J=$ $R r R r R J=R r R r J \subseteq R I=I$. This implies that $R r R J \subseteq I$. Thus, $r J \subseteq I$ and by Lemma [2.5, $(R / I)_{R}$ is endo-semiprime.

Now, the following result is immediate.

Corollary 2.8. The following conditions are equivalent:

(1) $R$ is a semiprime ring;

(2) $R_{R}$ is endo-semiprime;

(3) ${ }_{R} R$ is endo-semiprime.

Corollary 2.9. (1) $R_{R}$ is endo-cosemiprime if and only if every proper ideal of $R$ is semiprime.

(2) If $I$ is a right ideal in a ring $R$ such that $(R / I)_{R}$ is an endo-semiprime $R$-module, then $I$ behaves like a semiprime ideal, i.e., for any $a \in R, a R a \subseteq I$ concludes that $a \in I$.

(3) If $R_{R}$ is endo-cosemiprime, then $R$ is a semiprime ring.

(4) If $R_{R}$ is endo-cosemiprime, then $R_{R}$ is endo-semiprime.

Proof. (1) It follows from Proposition [2.6](2), by setting $I=0$.

(2) Note that $(R: I)_{l}=I$ and $(I: I)_{l} \subseteq(R: R)_{l}=R$. If $a R a \subseteq I$, where $a \in R$, then by Lemma [2.5, $a \in(R: I)_{l}=I$. 
(3) It follows from Corollary [2.6, because the zero ideal is a prime ideal in $R$.

(4) It follows from part (3) and Corollary 2.8 .

Remark 2.10. In Corollary [2.9, the converse of part (4) is not true in general. For example, $\mathbb{Z}_{\mathbb{Z}}$ is endo-semiprime because $\mathbb{Z}$ is a semiprime ring. But it is not endo-cosemiprime because $4 \mathbb{Z}$ is a proper ideal of $\mathbb{Z}$ that is not semiprime.

A right $R$-module $M$ is called retractable if for any nonzero submodule $N$ in $M$, $\operatorname{Hom}_{R}(M, N) \neq 0$ and $M_{R}$ is called epi-retractable if for any nonzero submodule $N$ in $M$, $\operatorname{Hom}_{R}(M, N)$ contains a surjective element.

A right $R$-module $M$ is called co-retractable if for any proper fully submodule $K$ in $M$, $\operatorname{Hom}_{R}(M / K, M) \neq 0$. Also, an $R$-module $M$ is called co-mono-retractable if for any proper submodule $K$ in $M, \operatorname{Hom}_{R}(M / K, M)$ contains an injective element; equivalently there exists a nonzero homomorphism $h \in \operatorname{End}\left(M_{R}\right)$ such that kerh $=K$. For more details see [4].

Proposition 2.11. Let $M_{R}$ be epi-retractable (resp., co-mono-retractable) such that $S=$ $\operatorname{End}\left(M_{R}\right)$ is a semiprime ring. Then $M_{R}$ is endo-semiprime (resp., endo-cosemiprime)

Proof. First suppose that $M_{R}$ is epi-retractable. Let $N$ be a nonzero fully invariant submodule of $M_{R}$ and $f S f N=0$, where $f \in S=\operatorname{End}\left(M_{R}\right)$. By assumption, there exists $0 \neq g \in S$ such that $g(M)=N$. Thus, $f S f g M=0$ and so $f g S f g M=0$. Since $S$ is semiprime, $f g=0$ and hence, $f g M=f N=0$.

For the second part, suppose that $M_{R}$ is co-mono-retractable. Let $K$ be a proper fully invariant submodule of $M$ and $f \in S=\operatorname{End}\left(M_{R}\right)$ such that $f S f(M) \subseteq K$. By assumption, there exists a nonzero homomorphism $h \in S$ such that kerh $=K$. Then $h \operatorname{Sh} f(M) \subseteq$ $h f S f(M) \subseteq h(K)=0$. Since $S$ is semiprime, $h f(M)=0$ and hence, $f(M) \subseteq \operatorname{ker} h=K$.

Remark 2.12. The epi-retractable property is required in Proposition [2.]1. For example, if $p$ is a prime number, then $\mathbb{Z}_{p^{\infty}}$ is not epi-retractable $\mathbb{Z}$-module and its endomorphism ring is the integral domain of $p$-adic integers that is a semiprime ring, but $\mathbb{Z}_{p^{\infty}}$ is not an endo-semiprime $\mathbb{Z}$ module. Because if $f$ is the homomorphism by multiplication $p$, then $f<\overline{1 / p^{2}}>\neq 0$ whereas $f^{2}<\overline{1 / p^{2}}>=0$.

Remark 2.10 , together with the following example show that the concepts of endo-semiprime and endo-cosemiprime are independent conditions.

Example 2.13. $\mathbb{Z}_{p^{\infty}}$ is an endo-cosemiprime $\mathbb{Z}$-module. Because for any proper submodule $K$ in $\mathbb{Z}_{p^{\infty}}, \mathbb{Z}_{p^{\infty}} / K \cong \mathbb{Z}_{p^{\infty}}$ as $\mathbb{Z}$-modules. Thus, $\mathbb{Z}_{p^{\infty}}$ is co-mono-retractable and so by Proposition [.]1, $\mathbb{Z}_{p^{\infty}}$ is endo-cosemiprime. However, by Remark [2.12, $\mathbb{Z}_{p^{\infty}}$ is not endo-semiprime. 
Alg. Struc. Appl. Vol. 5 No. 1 (2018) 69-80.

Corollary 2.14. Every semisimple $R$-module is both endo-semiprime and endo-cosemiprime.

Proof. Let $M$ be a semisimple $R$-module. Then $\operatorname{End}\left(M_{R}\right) \cong \oplus_{\alpha \in A} \mathbb{R} \mathbb{F} \mathbb{M}_{\Gamma_{\alpha}}\left(D_{\alpha}\right)$, for some suitable division ring $D_{\alpha}$ and nonempty set $\Gamma_{\alpha}$, where $\mathbb{R F M}_{\Gamma_{\alpha}}\left(D_{\alpha}\right)$ denotes a row finite $\Gamma_{\alpha^{-}}$ matrix ring over ring $D_{\alpha}$. We note that for any $\alpha \in A, \mathbb{R F} \mathbb{M}_{\Gamma_{\alpha}}\left(D_{\alpha}\right)$ is a prime ring and so $\operatorname{End}\left(M_{R}\right)$ is semiprime. Now, $M$ is endo-semiprime by Proposition 2.J]. On the other hand, since any semisimple $R$-module is co-mono-retractable, by Proposition [2.1], $M$ is endocosemiprime.

The following example indicates that an endo-prime module is not necessarily an endosemiprime module.

Example 2.15. Let $M=N_{1} \oplus N_{2}$ be a semisimple module such that simple submodules $N_{1}$ and $N_{2}$ are not isomorphic. By Corollary [2.14, $M$ is endo-semiprime but it is not endo-prime, because $\operatorname{End}\left(M_{R}\right) \cong \operatorname{End}\left(N_{1}\right) \oplus \operatorname{End}\left(N_{2}\right)$ is not prime.

Proposition 2.16. Let $M_{R}$ be an endo-semiprime $R$-module. If either $R$ is a commutative ring or $M$ is retractable, then $M$ is semiprime.

Proof. First assume that $R$ is commutative, $N$ is a nonzero submodule of $M$ and $a \in R$ such that $a^{2} \in \operatorname{ann}_{R}(N)$. We define $R$-homomorphism $f$ as follows:

$$
\begin{gathered}
f: M \rightarrow M \\
f(x)=x a .
\end{gathered}
$$

Then $f(S N)=S N a$ is a fully invariant submodule of $M$, where $S=\operatorname{End}\left(M_{R}\right)$. Thus, for any $h \in S$;

$$
f h f(S N)=f h(S N a)=f h(S N) a \subseteq f(S N) a=(S N a) a=S N a^{2}=0,
$$

and so $f S f(S N)=0$. Since $M_{R}$ is endo-semiprime and $S N$ is a fully invariant submodule of $M, \operatorname{ann}_{R}(S N)$ is semiprime and hence, $N a=0$, as desired.

Now, assume that $M$ is retractable and $N$ is a nonzero submodule of $M$ such that $N I^{2}=0$ and $N I \neq 0$, for some ideal $I$ of $R$. Then $S N I \neq 0$, where $S=\operatorname{End}\left(M_{R}\right)$. Since $M$ is retractable, there exists a nonzero homomorphism $f \in S$ such that $f(M) \subseteq S N I$. Therefore, for any $h \in S$;

$$
h f(M) \subseteq h(S N I)=h(S N) I \subseteq S N I .
$$

Hence;

$$
f h f(M) \subseteq f(S N I)=f(S N) I \subseteq f(M) I \subseteq(S N I) I=0 .
$$

Consequently, we have $f S f=0$ and since $M$ is endo-semiprime, $f=0$, a contradiction. Thus, $\operatorname{ann}_{R}(N)$ is semiprime. 
In [5], it is shown that if $M$ is an endo-prime $R$-module, then the fully invariant submodules of $M$ can not be summand. This fact is not true for endo-semiprime modules, because the $\mathbb{Z}$ modules $\mathbb{Z}_{6}$ is endo-semiprime and $3 \mathbb{Z}_{6}$ is a fully invariant submodule in $\mathbb{Z}_{6}$ with $\mathbb{Z}_{6}=2 \mathbb{Z}_{6} \oplus 3 \mathbb{Z}_{6}$.

The direct sum of two endo-semiprime modules may be not endo-semiprime. To see this, consider the following example.

Example 2.17. Let $p$ be a prime number. It is easy to see that $\mathbb{Z}$ and $\mathbb{Z}_{p}$ are endo-semiprime, as $\mathbb{Z}$-module. However, $\mathbb{Z} \oplus \mathbb{Z}_{p}$ is not endo-semiprime, because the ring

is not semiprime.

$$
\operatorname{End}\left(\left(\mathbb{Z} \oplus \mathbb{Z}_{p}\right)_{\mathbb{Z}}\right) \cong\left[\begin{array}{cc}
\mathbb{Z} & \mathbb{Z}_{p} \\
0 & \mathbb{Z}_{p}
\end{array}\right]
$$

In the following result we show that in some endo-semiprime modules, every fully invariant submodule is endo-semiprime.

Proposition 2.18. Let $M_{R}$ be an endo-semiprime $R$-module and $N$ be a fully invariant submodule of $M$. If either $N$ is a direct summand of $M$ or $M_{R}$ is quasi-injective, then $N$ is an endo-semiprime $R$-module.

Proof. If $N$ is a direct summand of $M$, then it is easy to check that $N$ is an endo-semiprime $R$ module. Now, assume that $M_{R}$ is quasi-injective and $K$ is a fully invariant submodule of $N$. Then $K$ is also a fully invariant submodule of $M$. We set $S=\operatorname{End}\left(N_{R}\right)$ and $\bar{S}=\operatorname{End}\left(M_{R}\right)$. Suppose that $f S f(K)=0$, for some $f \in S$. Then since $M$ is quasi-injective, there exists $\bar{f} \in \bar{S}$ such that $\left.\bar{f}\right|_{N}=f$. We show that $\bar{f} \bar{S} \bar{f}(K)=0$. For each $\bar{h} \in \bar{S}, h=\left.\bar{h}\right|_{N} \in S$ and since $K$ is a fully invariant submodule of $M$ we have:

$$
\bar{f} \bar{h} \bar{f}(K)=\bar{f} \bar{h} f(K)=\bar{f} h f(K)=f h f(K)=0 .
$$

Therefore, $\bar{f}=0$, because $M$ is endo-semiprime. So $f(K)=\left.\bar{f}\right|_{N}(K)=0$.

Theorem 2.19. Let $R$ be a ring. Consider the following statements:

(1) $R$ is semiprime.

(2) There exists a faithful retractable right (left) endo-semiprime $R$-module.

(3) All nonzero two-sided ideals of $R$ are endo-semiprime as right (left) $R$-modules.

Then $(1) \Leftrightarrow(2)$ and $(3) \Rightarrow(1)$. Moreover; if $R_{R}$ is injective, then $(1) \Rightarrow(3)$.

Proof. (1) $\Rightarrow(2)$. Let $I$ be a nonzero right ideal of $R$ and $0 \neq x \in I$. Then the map $f: R \rightarrow I$ defined by $f(r)=x r$ is a nonzero $R$-homomorphism. Thus, $R_{R}$ is retractable. Since $R$ is semiprime, by Corollary [2.8, $R_{R}$ is endo-semiprime. 
Alg. Struc. Appl. Vol. 5 No. 1 (2018) 69-80.

$(2) \Rightarrow(1)$. Let $M$ be a faithful retractable endo-semiprime right $R$-module. By Proposition [2.16, for any nonzero submodule $N$ in $M, \operatorname{ann}_{R}(N)$ is a semiprime ideal of $R$. Thus, $\operatorname{ann}_{R}(M)=0$ is also semiprime. Consequently, $R$ is a semiprime ring.

$(3) \Rightarrow(1)$ is trivial by Corollary [2.8.

$(1) \Rightarrow(3)$. Since $R$ is semiprime, by Corollary [2.8, $R_{R}$ is endo-semiprime. Now by Proposition [2.18, (3) is obtained because $R_{R}$ is injective.

Let $M$ be a right $R$-module. A nonzero submodule $N$ of $M$ is called essential in $M$, denoted by $N \leq_{e} M$, if $N \cap K \neq 0$, for any nonzero submodule $K$ of $M$. Also, the singular submodule of $M$ is the submodule $Z(M)=\left\{m \in M \mid \operatorname{ann}_{R}(m) \leq_{e} R_{R}\right\} . M$ is called singular (resp., nonsingular) if $Z(M)=M$ (resp., $Z(M)=0)$.

Remark 2.20. Let $N$ be a nonzero fully invariant submodule of $M$. If $M_{R}$ is nonsingular and $N \leq_{e} M$, then one can easily see that the restriction map $\varphi: \operatorname{End}\left(M_{R}\right) \rightarrow \operatorname{End}\left(N_{R}\right)$ is an injective homomorphism of rings, see [5, Lemma 1.8].

Proposition 2.21. Let $M$ be a quasi-injective nonsingular $R$-module and $N$ be an essential fully invariant submodule of $M$. Then $M_{R}$ is endo-semiprime if and only if $N_{R}$ is endosemiprime.

Proof. The necessity is covered by Proposition [2.18. For sufficiency, suppose that $N_{R}$ is endosemiprime and $K$ is a nonzero fully invariant submodule of $M$ such that $f S f(K)=0$, where $S=\operatorname{End}\left(M_{R}\right)$ and $f \in S$. By assumption, $N \leq_{e} M$ and so $N \cap K \neq 0$. Since both $N$ and $K$ are fully invariant, $N \cap K$ is also fully invariant. Now, as $M$ is quasi-injective and $f S f(N \cap K)=0$, we have $\left.\left.f\right|_{N} S^{\prime} f\right|_{N}(N \cap K)=0$, where $S^{\prime}=\operatorname{End}\left(N_{R}\right)$. Thus, $\left.f\right|_{N}(N \cap K)=0$ and so $\left.f\right|_{N \cap K}(N \cap K)=0$. Since $N$ is a fully invariant essential submodule of $M$, by Remark [2.20, $\varphi: \operatorname{End}(M) \rightarrow \operatorname{End}(N \cap K)$ is injective. Therefore, $\left.f\right|_{N \cap K}(N \cap K)=0$ implies that $f=0$ and so $f(K)=0$.

In the following example, we show that the concepts of semiprime and endo-semiprime are independent conditions.

Example 2.22. (a) Let $p$ be a prime number. By Example [2.77, the $\mathbb{Z}$-module $M=\mathbb{Z} \oplus \mathbb{Z}_{p}$ is not endo-semiprime. However we show that $M$ is a semiprime $\mathbb{Z}$-module. Let $0 \neq K \leq M$ and $I=n \mathbb{Z}$ is an ideal of $\mathbb{Z}$ such that $K I^{2}=0$. If $n=0$, then $K I=0$. Thus, suppose that $n \neq 0$ and $(x, y) \in K$. Then $(x, y) n^{2} \mathbb{Z}=0$ implies that $x n^{2}=0$ and $y n^{2}=0$; so $x=0$ and $p$ divides $y$ or $p$ divides $n$. In any case, we conclude that $(x, y) n \mathbb{Z}=0$. Thus, $K n \mathbb{Z}=0$, as desired. 
(b) Let $K$ be a field and $R=\left[\begin{array}{cc}K & K \\ 0 & K\end{array}\right], J=\left[\begin{array}{cc}0 & K \\ 0 & 0\end{array}\right]$ and $e=\left[\begin{array}{ll}1 & 0 \\ 0 & 0\end{array}\right]$. Since $J^{2}=0$, $R$ is not semiprime. Now set $M=e R$. Then $\operatorname{End}\left(M_{R}\right) \cong e R e \cong K$ as rings, and hence, $M$ is a semiprime left $K$-module. Thus, by Proposition [2.2, $M_{R}$ is endo-semiprime. On the other hand, it is easy to see that $\operatorname{ann}_{R}(M)=0$ and since $R$ is not a semiprime ring, we have $M_{R}$ is not semiprime.

Theorem 2.23. Both being endo-semiprime and being endo-cosemiprime are Morita invariant properties.

Proof. Suppose that $A$ and $B$ are Morita equivalent rings with inverse category equivalences $\alpha: \operatorname{Mod}_{A} \rightarrow \operatorname{Mod}_{B}$ and $\beta: \operatorname{Mod}_{B} \rightarrow \operatorname{Mod}_{A}$. First let $M$ be an endo-semiprime object in $\operatorname{Mod}_{A}$ and $N$ be a nonzero fully invariant submodule of $\alpha(M)$ with inclusion map $i$ to $\alpha(M)$. Then $\beta(i) \beta(N)$ is a nonzero submodule of $\beta \alpha(M)$. Now, assume that $h T h(N)=0$, for some $h \in T=\operatorname{End}\left(\alpha(M)_{B}\right)$. So for any $f$ and $f^{\prime}$ in $\operatorname{End}\left(M_{R}\right), h \alpha(f) h \alpha\left(f^{\prime}\right) i(N)=0$. Then $\beta(h) \beta \alpha(f) \beta(h) \beta \alpha\left(f^{\prime}\right) \beta(i)(\beta(N))=0$. Thus, $\beta(h) U \beta(h) U \beta(i)(\beta(N))=0$, where $U=$ $\operatorname{End}\left(\beta \alpha(M)_{A}\right)$. Since $\beta \alpha(M)$ is endo-semiprime and $U \beta(i)(\beta(N))$ is a nonzero fully invariant submodule of $\beta \alpha(M), \beta(h) U \beta(i)(\beta(N))=0$. Then $\beta(h) \beta(i)(\beta(N))=0$ and so $h(N)=0$.

Now, let $M$ be an endo-cosemiprime object in $\operatorname{Mod}_{A}$ and $N$ be a proper fully invariant submodule of $\alpha(M)$ with inclusion map $i$ to $\alpha(M)$. Then $\beta(i)(\beta(N))$ is a proper submodule of $\beta \alpha(M)$. We set $J=\operatorname{ann}_{U}(\beta \alpha(M) / \beta(i) \beta(N))$ where $U=\operatorname{End}\left((\beta \alpha(M))_{A}\right)$. Since $J \beta \alpha(M) \subseteq$ $\beta(i)(\beta(N)) \varsubsetneqq \beta \alpha(M)$, then $J \beta \alpha(M)$ is a proper fully invariant submodule of $\beta \alpha(M)$. We show that $\operatorname{ann}_{T}(\alpha(M) / N)$ is semiprime, where $T=\operatorname{End}\left(\alpha(M)_{B}\right)$. Let $h T h \alpha(M) \subseteq N$, for some $h \in T$. Then for any $f \in \operatorname{End}\left(M_{A}\right), h \alpha(f) h \alpha(M) \subseteq N=i N$. So $\beta(h) U \beta(h) \beta \alpha(M) \leq$ $\beta(i) \beta(N)$. Therefore, $\beta(h) U \beta(h) \leq J$. So $\beta(h) U \beta(h) \beta \alpha(M) \leq J \beta \alpha(M)$. Since $\beta \alpha(M)$ is endo-cosemiprime, then $\beta(h) \beta \alpha(M) \leq J \beta \alpha(M) \leq \beta(i) \beta(N)$ and so $h \alpha(M) \leq N$.

Now, we focus more on properties of endo-cosemiprime modules.

Proposition 2.24. If $R$ is a commutative ring and $M$ is an endo-cosemiprime $R$-module, then $R / \operatorname{ann}_{R}(M)$ is a semiprime ring.

Proof. We show that $\operatorname{ann}_{R}(M)$ is a semiprime ideal of $R$. Let $a \in R$ such that $a^{2} \in \operatorname{ann}_{R}(M)$. Then $f: M \rightarrow M$ defined by $f(x)=x a$ is an $R$-homoorphism. Now, we have $f S f(M)=$ $f S(M a) \subseteq f(M) a=M a^{2}=0$, where $S=\operatorname{End}\left(M_{R}\right)$. Since $M$ is endo-cosemiprime, $S$ is semiprime. Thus, $f(M)=0$ and so $M a=0$. 
Alg. Struc. Appl. Vol. 5 No. 1 (2018) 69-80.

Proposition 2.25. Let $M_{R}$ be a co-mono-retractable module. If either $M$ is nonsingular or every submodule of $M$ is a projective $R$-module, then $M$ is endo-cosemiprime.

Proof. First assume that $M$ is nonsingular and $N$ is a submodule of $M$. If $N$ is an essential submodule of $M_{R}$, then $(M / N)_{R}$ is singular. Since $M$ is co-mono-retractable, there exists a monomorphism $f: M / N \rightarrow M$. Then $M / N \cong f(M / N) \subseteq M$ and so $Z(M) \cap f(M / N)=$ $Z(f(M / N))$. Since $Z(M)=0$ and $Z(M / N)=M / N$, we have $f(M / N)=0$ and hence, $M / N=0$. Thus, $M=N$ and this implies that $M$ is semisimple; so it is endo-cosemiprime.

Now, suppose that every submodule of $M$ is a projective $R$-module and $N$ is a submodule of $M$. By assumption there exists a nonzero homomorphism $f \in \operatorname{End}\left(M_{R}\right)$ such that $\operatorname{ker} f=N$ and $M / N \cong \operatorname{Im} f$ is a projective submodule of $M$. Therefore, $0 \rightarrow N \rightarrow M \rightarrow M / N \rightarrow 0$ is a split short exact sequence, and so $M=N \oplus K$, for some submodule $K$ of $M$. Thus, $M$ is semisimple and by Corollary [2.14, it is endo-cosemiprime.

Proposition 2.26. Let $M_{R}$ be endo-cosemiprime and $S=\operatorname{End}\left(M_{R}\right)$, then $S_{S}$ is co-monoretractable if and only if $S$ is semisimple.

Proof. Let $S_{S}$ be co-mono-retractable. Since $M_{R}$ is endo-cosemiprime, $S$ is semiprime. So by [4, Corollary 1.7(7)], $S$ is a semisimple ring. The converse is straightforward.

Proposition 2.27. Let $R$ be a ring in which every two ideals are comparable. Then the followings are equivalent:

(1) $\operatorname{ann}_{R}(M)$ is semiprime;

(2) $\operatorname{ann}_{R}(K)=\operatorname{ann}_{R}(M)$ or $\operatorname{ann}_{R}(M / K)=\operatorname{ann}_{R}(M)$, for any nontrivial submodule $K$ of $M$;

(3) $\operatorname{ann}_{R}(K)=\operatorname{ann}_{R}(M)$ or $\operatorname{ann}_{R}(M / K)=\operatorname{ann}_{R}(M)$, for any nontrivial fully invariant submodule $K$ of $M$.

Proof. (1) $\Rightarrow(2)$. Let $K$ be a nontrivial submodule of $M$. By assumption, $\operatorname{ann}_{R}(K) \subseteq$ $\operatorname{ann}_{R}(M / K)$ or $\operatorname{ann}_{R}(M / K) \subseteq \operatorname{ann}_{R}(K)$. If $\operatorname{ann}_{R}(K) \subseteq \operatorname{ann}_{R}(M / K)$, then $\left(\operatorname{ann}_{R}(K)\right)^{2} \subseteq$ $\operatorname{ann}_{R}(M)$. For any $x \in \operatorname{ann}_{R}(K)$, we have $(x R)^{2} \subseteq\left(\operatorname{ann}_{R}(K)\right)^{2} \subseteq \operatorname{ann}_{R}(M)$. Since by (1), $\operatorname{ann}_{R}(M)$ is semiprime, $x R \subseteq \operatorname{ann}_{R}(M)$ and so $x \in \operatorname{ann}_{R}(M)$. Thus, $\operatorname{ann}_{R}(K)=\operatorname{ann}_{R}(M)$. The other case is similar.

$(2) \Rightarrow(3)$ is trivial.

$(3) \Rightarrow(1)$ Let $I$ be an ideal of $R$ such that $M I^{2}=0$. If $M I=M$, or $M I=0$, then $M I^{2}=M I=0$. Thus, we assume that $M I$ is a nontrivial submodule of $M$. It is clear that $M I$ is fully invariant. By $(3), \operatorname{ann}_{R}(M I)=\operatorname{ann}_{R}(M)$ or $\operatorname{ann}_{R}(M / M I)=\operatorname{ann}_{R}(M)$. If $\operatorname{ann}_{R}(M I)=\operatorname{ann}_{R}(M)$, then $I \subseteq \operatorname{ann}_{R}(M I)=\operatorname{ann}_{R}(M)$ and so $M I=0$. If $\operatorname{ann}_{R}(M / M I)=$ 
$\operatorname{ann}_{R}(M)$, then $I \subseteq \operatorname{ann}_{R}(M / M I)=\operatorname{ann}_{R}(M)$. Thus, in any case, $M I^{2}=M I=0$, as desired.

Corollary 2.28. Let $R$ be a ring in which every two ideals are comparable and $M$ be a faithful $R$-module. Then the following statements are equivalent:

(1) $R$ is a semiprime ring;

(2) $\operatorname{ann}_{R}(K)=0$ or $\operatorname{ann}_{R}(M / K)=0$, for any nontrivial submodule $K$ of $M$;

(3) $\operatorname{ann}_{R}(K)=0$ or $\operatorname{ann}_{R}(M / K)=0$, for any nontrivial fully invariant submodule $K$ of $M$.

\section{ACKNowledgments}

The authors would like to thank the referees for helpful comments and suggestions that improved this paper.

\section{REFERENCES}

[1] M. Behboodi and S. H. Sojaee, On chains of classical prime submodules and dimensions theory of modules, Bull. Iranian Math. Society, 36(1) (2010), 149-166.

[2] S. Ceken, M. Alkan and P. F. Smith, Second modules over noncommutative rings, Comm. Algebra, 41 (2013), 83-98.

[3] J. Dauns, Prime modules, J. Reine Angew. Math., 298 (1978), 156-181.

[4] A. Ghorbani, Co-epi-retractable modules and co-pri rings, Comm. Algebra, 38 (2010), 3589-3596.

[5] A. Haghany and M. R. Vedadi, Endoprime modules, Acta Math. Hungar., 106(1-2) (2005), 89-99.

[6] B. Sarac, On semiprime submodules, Comm. Algebra, 37(7) (2009), 2485-2495.

[7] R. Wisbauer, Foundations of module and ring theory, Gordon and Breach Science Publishers Reading (1991).

[8] S. Yassemi, The dual notion of prime submodules, Arch. Math. Brno., 37 (2001), 273-278.

\section{Parvin Karimi Beiranvand}

Department of mathematics, Lorestan university,

P.O.Box 465, Khoramabad, Iran.

karimi.pa@fs.lu.ac.ir

\section{Reza Beyranvand}

Department of mathematics, Lorestan university, P.O.Box 465, Khoramabad, Iran.

beyranvand.r@lu.ac.ir; beyranvand.r94@gmail.com 